Y. Furuta

Nagoya Math. J.

Vol. 66 (1977), 167-182

\title{
NOTE ON GLASS NUMBER FACTORS AND PRIME DECOMPOSITIONS
}

\author{
YOSHIOMI FURUTA
}

\section{Introduction}

Let $K$ be a Galois extension of an algebraic number field $k$ of finite degree with Galois group $g$, D be a congruent ideal class group of $K$, and $M$ be the class field over $K$ corresponding to $\mathfrak{D}$. Assume that $M$ is normal over $k$. Then $g$ acts on $D$ as a group of automorphisms. Donote by $I_{g}$ the augmentation ideal of the group ring $Z_{g}$ over the ring of integers $Z$. Then we have a sequence $\mathfrak{D} \supset I_{g} \mathfrak{D} \supset I_{g}^{2} \mathfrak{D} \supset \cdots$ and a sequence of the corresponding class fields $K=K_{M / k}^{(0)} \subset K_{M / k}^{(1)} \subset K_{M / k}^{(2)} \subset \cdots$. We call $K_{M / k}^{(i)}$ the $i$-th central class field of $K$ in $M$ with respect to $k$. We put simply $K^{(i)}=K_{M / k}^{(i)}$, when it is not in danger of confusion.

In the previous paper [10], we have shown that the Galois group $G\left(K^{(i+1)} / K^{(i)}\right)$ is isomorphic to a factor group of $G\left(K^{(1)} / K\right)$ or of slightly modified group of $G\left(K^{(1)} / K\right)$ when $K$ is non-cyclic over $k$.

In the present paper we apply the above result firstly to the case where $K$ is cyclic over $k$ and we have more explicit structure of $G\left(K^{(i+1)} / K^{(i)}\right)$. In fact we have a formula of the extension degree of $K^{(i+1)} / K^{(i)}$ in $\S 2$, which generalize the genus formula in [8] when $K$ is cyclic over $k$. Furthermore in $\S 3$ we express the structure of $G\left(K^{(i+1)} / K^{(i)}\right)$ by using "Auflösung" characters of H. W. Leopoldt [19], when the ground field $k$ is the rational number field $\boldsymbol{Q}$.

Secondly we study on prime decompositions in $K^{(i+1)} / K^{(i)}$ and in $\S 5$ we have explicit criteria of prime decompositions for some non-abelian extensions. As a special case we have a new expression of the reciprocity of the biquadratic residue symbol. $\S 2$ and $\S 3$ are unnecessary to the argument of $\S 4$ and $\S 5$.

Received June 30, 1976. 


\section{§1. Preliminaries}

1.1. Notation being as in Introduction, suppose that $K$ is a cyclic extension of $k$, whose Galois group is $g$ generated by $\sigma$. Then for $i=$ $0,1,2, \cdots$, we have easily ${ }^{1)}$

$$
G\left(K^{(i+1)} / K^{(i)}\right) \cong \mathfrak{D}^{(o-1)^{i}} / \mathfrak{D}^{(o-1)^{i+1}} \cong \mathfrak{D} /\left(\Re^{(i)} \mathfrak{D}^{\sigma-1}\right),
$$

where $\Re^{(i)}=\Re^{(i)}(\mathfrak{D})$ is the group of elements $\mathfrak{c}$ of $\mathfrak{D}$ such that $\mathfrak{c}^{(\sigma-1)^{i}}=1$. Since $K$ is cyclic over $k$, the field $K^{(1)}$ coincides with the genus field $K^{*}=K_{M / k}^{*}$ of $K$ in $M$, which is by definition the maximal extension of $K$ contained in $M$ and obtained by composing an abelian extension over k. We have ${ }^{2)}$

$$
\mathfrak{D} / \mathfrak{D}^{\sigma-1} \cong G\left(K^{(1)} / K\right)=G\left(K^{*} / K\right) \cong H(K / k) /\left(k^{\times} N_{K / k} H(M / K)\right),
$$

where $H(M / K)$ and $H(K / k)$ are the idele group of $K$ and $k$ corresponding to $M$ and $K$ respectively, and $k^{\times}$is the principal idele group of $k$.

Denote by $\mathfrak{S}(K / k)$ and $\mathfrak{S}^{*}(K / k)$ the congruent ideal group of $k$ corresponding to $H(K / k)$ and $k^{\times} N_{K / k} H(M / k)$ respectively. Then we have

$$
G\left(K^{*} / K\right) \cong \mathfrak{S}(K / k) / \mathfrak{S}^{*}(K / k) \text {. }
$$

Denote by $\mathscr{S}(M / K)$ the congruent ideal group of $K$ corresponding to $M$ and by $\mathfrak{\Im}=\mathscr{\Im}(M / K)$ the conductor of $\mathscr{S}_{(}(M / K)$. Let $\Re_{0}^{(i)}$ be the group of ideals $\mathfrak{a}$ of $k$ such that $a=N_{K / k} \mathfrak{A}$ for some ideal $\mathfrak{A} \in \mathfrak{\Re}^{(i)}$. Then (1), (2) and (3) imply

$$
G\left(K^{(i+1)} / K^{(i)}\right) \cong \mathscr{S}(K / k) / \Re_{0}^{(i)} \mathfrak{S}^{*}(K / k) .
$$

1.2. The structure of $\mathfrak{S}^{*}(K / k)$ is known for many cases explicitly. ${ }^{3)}$ In the following we shall show a recursive method to get $\Re^{(i)}$ or $\AA_{0}^{(i)}$. In order to determine $\AA^{(i)}$, it is enough to get a finite number of integral ideals by which all classes of $\AA^{(i)}$ are represented $\bmod \mathfrak{S}(M / K)$. We call a system of these ideals a full set of representatives of $\AA^{(i)}$. We call the set of norms of these ideals to $k$ a full set of representatives of $\AA_{0}^{(i)}$.

Suppose that for $\nu=1, \cdots, t_{i}$, ideals $\mathfrak{A}_{\nu}$ consist a full set of representatives of $\AA^{(i)}$. When $N_{K / k} \mathfrak{A}_{\nu} \in N_{K / k} \mathfrak{S}(M / K)$, we can choose an integral ideal $\mathfrak{B}_{\nu}$ of $K$ such that

1) See Y. Furuta $[10, \S 1]$.

2) Cf. Y. Furuta $[10, \S 2]$.

3) See for instance H. W. Leopoldt [19], A. Fröhlich [5] and Y. Furuta [8]. 


$$
\mathfrak{B}_{\nu}^{\sigma-1} \equiv \mathfrak{H}_{\nu} \bmod . \mathfrak{S}_{\mathcal{C}}(M / K) \text {. }
$$

Then it follows from the definition of $K^{(i)}$ that $\Re_{\nu}$ for $\nu=1, \cdots, t_{i}$ and $B_{\nu}$ chosen as above make a full set of representatives of $\Re^{(i+1)}$.

Remark. Since the above $\mathfrak{H}_{\nu}$ and $\mathfrak{B}_{\nu}$ are representatives mod. $\mathfrak{S}(M / K)$, we can restrict them to be prime ideals of $K$ of absolute degree 1 . When that is the case, we call the set of $\mathfrak{A}_{\nu}$ and $\mathfrak{B}_{\nu}$ resp. $\mathfrak{a}_{\nu}=N_{K / k} \mathfrak{A}_{\nu}$ and $\mathfrak{b}_{\nu}$ $=N_{K / k} \mathfrak{B}_{\nu}$ a full set of prime representatives of degree 1 of $\AA^{(i)}$ resp. of $\AA_{0}^{(i)}$.

1.3. In the case where $k$ is the rational number field $Q, K$ is a quadratic field and $M$ is a ray class field over $K$ mod. $\widetilde{F}$ for some divisor $\widetilde{F}$ of $K$, we have further the following way ${ }^{4}$ of the determination of $\Re_{0}^{(i+1)}$ from $\Re_{0}^{(i)}$. We assume, by the above remark, that the representatives of $\AA^{(i)}$ are prime ideals of $K$ of degree 1 . Let $\mathfrak{p}$ be such a representative and assume $p=N_{K / Q} \mathfrak{p} \in N_{K / Q} S_{K}(\mathfrak{F}), S_{K}(\mathfrak{F})$ being the ray $\bmod . \widetilde{\gamma}$ in $K$. Then there exists a prime ideal $\mathfrak{q}$ of $K$ of degree 1 such that $\mathfrak{q}^{\sigma-1} \equiv \mathfrak{p} \bmod . S_{K}(\mathfrak{l})$. Hence for some $(\alpha) \in S_{K}(\mathfrak{⿰})$ we have

$$
\alpha=\mathfrak{p q} \mathfrak{q}^{1-\sigma}=\mathfrak{p q}^{2} / N_{K / Q} \mathfrak{q}=\beta / q,
$$

where $\sigma$ is the non-trivial automorphism of $K$ over $\boldsymbol{Q}, q=N_{K / \mathcal{Q}} q$, the rational prime, and $\beta$ is an integer of $K$ such that $q \equiv \beta$ mod. $\widetilde{\mho}$. Since $N_{K / Q} \alpha=N_{K / Q} \mathfrak{p}=p$, we have

$$
p q^{2}=N_{K / Q} \beta .
$$

Conversely let $p \in{\Re_{0}^{(i)}}^{(i . e .,} p=N_{K / \mathbb{q}} \mathfrak{p}$ and $\mathfrak{p} \in \Re^{(i)}$. Let $q$ be a rational prime which satisfies (6) for some integer $\beta$ of $K$ such that $q \equiv$ $\beta$ mod. $\widetilde{\gamma}, \beta$ has no rational integral divisor, and $q$ be decomposed completely in $K$. Then (6) implies $\beta=\mathfrak{p q}^{2}, N_{R / q} \mathfrak{q}=q$, hence $\mathfrak{p q}^{1-\sigma}=\beta / q \in$ $S_{K}(\widetilde{\mho})$, which means $q \in \Re_{0}^{(i+1)}$.

Now let $D$ be the discriminant of $K$. Then the above $q$ is a primitive solution $z$ of the Diophantine equation

$$
x^{2}-D y^{2}-4 p z^{2}=0 \text {. }
$$

Therefore a full set of prime representatives of degree 1 of $\AA_{0}^{(i+1)}$ is obtained from the full set of prime representatives $p$ of degree 1 of

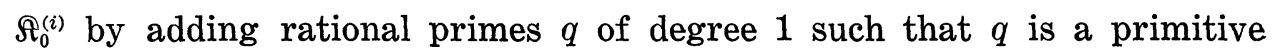

4) Cf. H. Hasse [14] and G. Gras [12, §IV, B]. 
solution of (7) by $z=q$ and $\frac{1}{2}(x+y \sqrt{D}) \equiv q \bmod . \widetilde{\mho}$.

\section{§2. Nilpotent factors of the ideal class group of cyclic extensions}

2.1. We can study the structure of the ideal class group of cyclic extensions of any degree by using (1). This is a generalization of $\mathrm{H}$. Hasse [14] and G. Gras [12] by means of dual way ${ }^{5)}$ in some sense.

For the sake of simplicity we treate of the case where $M$ is the absolute class field of $K$, in wide sense or in narrow sense, which we denote by $K^{\#}$.

For an algebraic number field $K$, we denote by $J_{K}$ the idele group, by $K^{\times}$the principal idele group and by $U_{K}$ the unit idele group of $K$ whose real infinite components are of all non-zero real numbers or positive real numbers according as we treate on the absolute class field in wide sense or in narrow sense. Denote by $K_{\Re}^{\times}$the multiplicative group of non-zero elements of the completion $K_{\mathfrak{B}}$ of $K$ at $\mathfrak{\beta}$, and by $U_{\mathfrak{\beta}}$ the unit group of $K_{\mathfrak{\beta}}$, which are embedded in $J_{K}$ in usual way.

Now let $K / k$ be a cyclic extension and let $M=K^{\sharp}$. Let $R^{(i)}$ be a full set of representatives of $\Omega^{(i)}$ and assume ${ }^{6)}$ that $R^{(i)}$ is consisted by prime ideals of degree 1 over $k$. Let $R_{0}^{(i)}$ be the set of all $N_{K / k} \Re$ where $\mathfrak{\beta} \in R^{(i)}$. Denote further by $K_{i}^{*}$ the class field over $K$ corresponding to $\Re^{(i)} \mathfrak{D}^{\sigma-1}$. Then $K_{i}^{*}$ is the maximal extension over $K$ which is contained in $K^{*}$ and in which all primes of $R^{(i)}$ are completely decomposed. We put

$$
H^{(i)}=\prod_{p \in R_{0}^{(i)}}\left(H \cap H^{*} k_{p}^{\times}\right),
$$

where $H=H(K / k)$ is the subgroup of $J_{k}$ corresponding to $K$ as in $\S 1.1$, and $H^{*}=k^{\times} N_{K / k} U_{K}$. Then (1) implies

$$
G\left(K^{(i+1)} / K^{(i)}\right) \cong G\left(K_{i}^{*} / K\right) \cong H / H^{(i)} .
$$

Moreover by [8, Proposition 2], we have $H^{*}=k^{\times} \prod_{\mathfrak{p}}\left(H \cap U_{\mathfrak{p}}\right)$ where $\mathfrak{p}$ runs over all primes of $k$. Hence by simple calculations, (8) implies

$$
\begin{aligned}
H^{(i)} & =k^{\times} \cdot \prod_{p \notin R_{0}^{(i)}}\left(H \cap U_{p}\right) \cdot \prod_{p \in R_{0}^{(i)}}\left(H \cap k_{p}^{\times}\right) \\
& =k^{\times} \prod_{p \in R_{0}^{(i)}}\left(H \cap U_{p}\right) \cdot \prod_{p \in R_{0}^{(i)}} k_{p}^{\times} .
\end{aligned}
$$

5) The investigation in this way has been treated in some cases by E. Inaba [15], A. Fröhlich [3] and H. Koch [17].

6) See Remark of $\S 1.2$. 
Now (9) and (10) imply the following

Proposition 2.1. Let $K$ be a cyclic extension of $k$, and $K^{(i)}$ be the $i$-th central class field of $K$ with respect to $k$ in the absolute class field $K^{\#}$ of $K$. Let $R_{0}^{(i)}$ be a full set of representatives of $\Re_{0}^{(i)}$ which consists of primes of $k$ of degree 1 in $K$. Then we have

$$
G\left(K^{(i+1)} / K^{(i)}\right) \cong H /\left(k^{\times} \cdot \prod_{p}\left(H \cap U_{p}\right) \cdot \prod_{p \in R_{0}^{(i)}} k_{p}^{\times}\right),
$$

where $H=H(K / k)$, the idele group of $k$ corresponding to $K$.

2.2. Notation being as above, denote further by $z_{K / k}^{(i)}$ the extension degree $\left(K^{(i)}: K^{(i-1)}\right)$ for $i \geqq 1$, where $K^{(0)}=K$. We call $z_{K / k}^{(i)}$ the $i$-th central class number of $K$ with respect to $k$. Now by an analogous calculation as the genus formula in $\left[8\right.$, Theorem $\left.{ }^{7)}\right]$ we have a formula for $z_{k / k}^{(i)}$ as follows. By (9) we have

$$
z_{K / k}^{(i)}=\left(K^{(i)}: K^{(i-1)}\right)=\left(K_{i-1}^{*}: K\right)=\left(K_{i-1}^{*}: k\right) /(K: k) .
$$

For a wile denote by $p$ resp. $q$ primes of $k$ contained resp. not contained in $R_{0}^{(i-1)}$. Then

$$
\left(K_{i-1}^{*}: k\right)=\left(J_{k}: H^{(i-1)}\right)=\left(J_{k}: k^{\times} \prod_{q} U_{q} \prod_{p} k_{p}^{\times}\right) \cdot\left(k^{\times} \prod_{q} U_{q} \prod_{p} k_{p}^{\times}: H^{(i-1)}\right) .
$$

Denote by $\mathfrak{P}$ resp. $\mathfrak{Q}$ one of prime divisors of $\mathfrak{p}$ resp. $\mathfrak{q}$ in $K$ fixed once for all. Denote for a wile by $N$ the norm for local fields. Then since $\mathfrak{p}$ is decomposed completely in $K$ by the assumption, we have

$$
k^{\times} \prod_{q} N U_{a} \prod_{p} N K_{\mathfrak{p}}^{\times} \cap \prod_{q} U_{q} \prod_{p} k_{p}^{\times}=\left(k^{\times} \cap \prod_{q} U_{q} \prod_{p} k_{p}^{\times}\right) \cdot \prod_{q} N U_{\mathfrak{a}} \prod_{p} N K_{\mathfrak{p}}^{\times} \text {. }
$$

Moreover since $N K_{\mathfrak{p}}^{\times}=k_{\mathfrak{p}}^{\times}$, we have

$$
\begin{aligned}
& \left(k^{\times} \prod_{\mathfrak{q}} U_{\mathfrak{a}} \prod_{\mathfrak{p}} k_{\mathfrak{p}}^{\times}: H^{(i-1)}\right)=\left(k^{\times} \prod_{\mathfrak{q}} U_{\mathfrak{a}} \prod_{\mathfrak{p}} k_{\mathfrak{p}}^{\times}: k^{\times} \prod_{\mathfrak{q}} N U_{\mathfrak{a}} \prod_{\mathfrak{p}} N K_{\mathfrak{P}}^{\times}\right) \\
& =\left(\prod_{q} U_{q} \prod_{p} k_{p}^{\times}: k^{\times} \prod_{q} N U_{\mathfrak{Q}} \prod_{\mathfrak{p}} N K_{\mathfrak{p}}^{\times} \cap \prod_{q} U_{q} \prod_{p} k_{p}^{\times}\right) \\
& =\frac{\prod_{\mathfrak{a}}\left(U_{\mathrm{q}}: N U_{\mathfrak{a}}\right)}{\left(\left(k^{\times} \cap \prod_{\mathfrak{a}} U_{\mathrm{q}} \prod_{\mathfrak{p}} k_{\mathfrak{p}}^{\times}\right):\left(k^{\times} \cap \prod_{\mathrm{q}} N U_{\mathfrak{Q}} \prod_{p} N K_{\mathfrak{p}}^{\times}\right)\right)} \text {. }
\end{aligned}
$$

Now we have the following

7) Cf. also L. Goldstein [11, §2]. 
Proposition 2.2. Let $K$ be a cyclic extension of $k$. For a prime $\mathfrak{q}$ of $k$, denote by $e_{\mathrm{q}}$ the ramification index of $\mathfrak{q}$ in $K$. Let $R_{0}^{(i)}$ be a full set of representatives of $\AA_{0}^{(i)}$ which consists of primes of degree 1 in $K$. Denote by $E\left(R_{0}^{(i)}\right)$ the group of all $R_{0}^{(i)}$-units ${ }^{8)}$ of $k$, and by $\tilde{E}\left(R_{0}^{(i)}\right)$ the group of all elements of $E\left(R_{0}^{(i)}\right)$ which are everywhere locally norm from $K$. Denote further by $h\left(R_{0}^{(i)}\right)$ the extension degree of the maximal extension over $k$ which is contained in the absolute class field of $k$ and in which all primes of $R_{0}^{(i)}$ are decomposed completely. Then we have

$$
z_{K / k}^{(i+1)}=\frac{h\left(R_{0}^{(i)}\right) \prod_{q \in R_{0}^{(i)}} e_{q}}{(K: k)\left(E\left(R_{0}^{(i)}\right): \tilde{E}\left(R_{0}^{(i)}\right)\right)} .
$$

Remark. For $i=0$, the above formula coinsides with the genus formula in [8] or in L. Goldstein [11], when $K$ is cyclic over $k$.

\section{§3. Nilpotent factors of the ideal class group of cyclic extensions over $\boldsymbol{Q}$}

3.1. When $K$ is an abelian extension over the rational number field $\boldsymbol{Q}$ and $M$ is the absolute class field of $K$ in narrow sense, the genus field $K^{*}=K_{M / k}^{*}$ corresponds to the "Auflösung" character group by H. W. Leopoldt [19]. Thus by (1) and (2), we can study the structure of $G\left(K^{(i+1)} / K^{(i)}\right)$ by means of the above character group.

For an abelian extension $K / \boldsymbol{Q}$, let $X$ be the corresponding character group, namely the character group of the congruent ideal class group corresponding to $K$, which is also the character group of $G(K / Q)$ via Artin's reciprocity. For a character $\chi$ in $X$ denote by $K_{x}$ the abelian field corresponding to $\chi$. The $p$-component $\chi_{p}$ of $\chi$ for a rational prime $p$ is defined by

$$
\chi_{p}(a)=\chi\left(\frac{|a|, K_{x}}{p}\right)
$$

when $a \in \boldsymbol{Q}$ and is prime to the conductor of $\chi$. Then $\chi_{p}$ is a character of some congruent ideal class group. The "Auflösung" $X^{*}$ of $X$ is the group generated by all $\chi_{p}$ where $\chi \in X$ and $p$ runs over all rational primes. H. W. Leopoldt [19] has proved.

$$
G\left(K^{*} / K\right) \cong X^{*} / X
$$

8) This means elements of $k$ whose prime divisors are at most primes contained in $R_{0}^{(i)}$; and which are totally positive when $K^{\#}$ is the absolute class field in narrow sense. 
3.2. Put $H^{*}=\boldsymbol{Q}^{\times} \cdot N_{K / Q} U_{K}$. Then it follows from [8, Proposition 1] that

$$
G\left(K^{*} / K\right) \cong H / H^{*},
$$

where $H=H(K / Q)$. For the sake of convenience we shall imply ${ }^{9}$ Leopoldt's formula (11) from (12). Since we treate here the absolute class field in narrow sense, we have to take the group of all positive real numbers as the infinite component $U_{p_{\infty}}$ of $U_{\boldsymbol{Q}}$. Then $J_{\boldsymbol{Q}}=\boldsymbol{Q}^{\times} U_{\boldsymbol{Q}}$ and an element $\mathfrak{a}$ of $J_{Q}$ is expressed uniquely as $\mathfrak{a}=a \mathfrak{u}$, where $a \in \boldsymbol{Q}^{\times}$and $\mathfrak{u}=$ $\left(u_{p}\right) \in U_{Q}$. Let $H_{\chi}$ be the subgroup of $J_{Q}$ corresponding to $K_{\chi}$, and denote by $S_{0}$ the set of all finite primes of $\boldsymbol{Q}$. Then we have

$$
\begin{aligned}
H^{*} & =\boldsymbol{Q}^{\times} \prod_{p \in S_{0}}\left(H \cap U_{p}\right) \cdot U_{p_{\infty}}=\boldsymbol{Q}^{\times} \cdot \prod_{p \in S_{0}}\left(\left(\bigcap_{\chi \in X} H_{\chi}\right) \cap U_{p}\right) \cdot U_{p_{\infty}} \\
& =\bigcap_{\substack{\chi \in X \\
p \in S_{0}}} \boldsymbol{Q}^{\times} \prod_{\substack{q \neq p \\
q \in S_{0}}} U_{q} \cdot\left(H_{\chi} \cap U_{p}\right) \cdot U_{p_{\infty}} .
\end{aligned}
$$

For $\chi \in X$ and $p \in S_{0}$ we define $\varphi_{x, p}$ by

$$
\varphi_{\chi, p}(\mathfrak{a})=\varphi_{\chi, p}(a \mathfrak{u})=\left(\frac{u_{p}, K_{\chi}}{p}\right),
$$

where $\mathfrak{a}=a \mathfrak{u} \in J_{\boldsymbol{Q}}, \quad a \in \boldsymbol{Q}^{\times}, \mathfrak{t}=\left(u_{p}\right) \in U_{\boldsymbol{Q}}$. Then $\varphi_{x, p}$ is a differential of $J_{Q}$ in the sense of the class field theory. The kernel of $\varphi_{x, p}$ is equal to $\boldsymbol{Q}^{\times} \prod_{q \neq p, q \in S_{0}} U_{q} \cdot\left(H_{\chi} \cap U_{p}\right) \cdot U_{p_{\infty}}$, and it follows from (13) that the character group of $J_{Q} / H^{*}$ is generated by $\varphi_{x, p}$ where $\chi \in X$ and $p \in S_{0}$. Let $\psi_{x, p}$ be the congruent ideal character corresponding to $\varphi_{x, p}$. Then since the conductor of $\varphi_{x, p}$ is equal to the product of a power of $p$ and $p_{\infty}$, we see

$$
\psi_{\chi}, p(a)=\chi\left(\frac{a, K_{x}}{p}\right)^{-1}=\chi_{p}^{-1}(a),
$$

for $a \in \boldsymbol{Q}$ which is positive and prime to the conductor of $\chi$. Hence $X^{*}$ corresponds to $K^{*}$ and (11) is implied.

3.3. Assume that $K$ is cyclic extension over $k=\boldsymbol{Q}$. Notation $\AA^{(i)}$, $R^{(i)}, R_{0}^{(i)}$ and $K_{i}^{*}$ be as in $\S 2.1$. For the character group $X$ of $K / \boldsymbol{Q}$ put

$$
X_{i}^{*}=\left\{\chi \in X^{*} \mid \chi(p)=1 \quad \text { for all } p \in R_{0}^{(i)}\right\} \text {. }
$$

Then by the definition of $K_{i}^{*}$, the character group corresponding $K_{i}^{*}$ is equal to $X_{i}^{*} X$. Now we have

9) Cf. $[8$, Remark]. 
Proposition 3.1. Let $K$ be a cyclic extension over $\boldsymbol{Q}, X$ be the character group for $K / \mathbf{Q}$, and $K^{(i)}$ be the $i$-th central class field of $K$ with respect to $\boldsymbol{Q}$ in the absolute class field of $K$ in narrow sense. Then $X_{i}^{*}$ being as (14), we have

$$
G\left(K^{(i+1)} / K^{(i)}\right) \cong X_{i}^{*} X / X \cong X_{i}^{*} /\left(X_{i}^{*} \cap X\right) .
$$

3.4. In the case where $K=\boldsymbol{Q}(\sqrt{d})$, a quadratic field, we can determine $X_{i}^{*}$ as follows more explicitly by using the result of $\S 1.3$.

Since $\AA^{(1)}$ consists of ambigous classes and any ambigous class of $\boldsymbol{Q}(\sqrt{d})$ is represented by a ramified prime idea ${ }^{10)}, R_{0}^{(1)}$ consists of prime divisors of the discriminant $D$ of $\boldsymbol{Q}(\sqrt{\bar{d}}) / \boldsymbol{Q}$.

Let $D=q_{1}^{*} \cdots q_{n}^{*}$ be the decomposition of $D$ to prime discriminants, namely $q^{*}=(-1)^{(q-1) / 2} q$ for a prime $q \neq 2$ and $q^{*}=-4$ or \pm 8 for $q=2$. Put

$$
\chi_{q^{*}}(a)=\left(\frac{q^{*}}{a}\right) .
$$

Then $^{11)} X^{*}$ is generated by $\chi_{q_{1}^{*}}, \cdots, \chi_{q_{r}^{*}}$ Let $p$ be a prime number such that ${ }^{12)} \chi_{q_{t}^{*}}(p)=1$ for $i=1, \cdots, r$. Assume further that the Diophantine equation

$$
x^{2}-D y^{2}-4 p=0
$$

has no solution. Then by $\S 1.3$ the Diophantine equation

$$
x^{2}-D y^{2}-4 p z^{2}=0
$$

has a primitive solution $x, y, z$, where $z$ can be taken as a prime number. We call it a primitive prime solution of (17). Then a full set of representatives $R_{0}^{(i+1)}$ is determined from $R_{0}^{(i)}$ recursively as follows. Let $S_{0}^{(i)}$ be a subset of $R_{0}^{(i)}$ which consists of rational primes $p \in R_{0}^{(i)}$ such that $\chi_{q_{i}^{*}}(p)=1$ for $i=1, \cdots, r$, and (16) has no solution. For every such $p$ choose a primitive prime solution $p^{\prime}=z$ of (17). Then as $R_{0}^{(i+1)}$ we can take the union of primes $p^{\prime}$ and $R_{0}^{(i)}$.

Then we can determine $X_{i}^{*}$ by (14), and $z_{K / k}^{(i)}$ by Proposition 2.2 explicitly.

10) See for instance G. Gras [12, Corollaire 4.2].

11) See also H. Hasse [13].

12) We set always $\chi_{q^{*}}(q)=1$. 


\section{§4. $E L$-genus central extensions}

4.1. Let $K$ be a Galois extension of an algebraic number field $k$ of finite degree. We call an extension $K^{\prime}$ of $K$ a central extension of $K$ with respect to $k$, when $K^{\prime}$ is normal over $k$ and the Galois group of $K^{\prime}$ over $K$ is contained in the center of the Galois group of $K^{\prime}$ over $k$. We call $K^{\prime}$ an EL-genus extension of $K$ with respect to $k$, when each local completion of $K^{\prime}$ is equal to the composite of the corresponding local completion of $K$ and an abelian extension of the corresponding local completion of $k$. For an extension $M$ of $K$, we denote by $\hat{K}_{M / k}$ the maximal extension of $K$ which is contained in $M$ and is a central $E L$ genus extension of $K$ with respect to $k$. We denote further by $K_{M / k}^{*}$ the genus field of $K$ in $M$ with respect to $k$. The structure of the Galois group $G\left(K_{M / k}^{*} / K\right)$ and $G\left(\hat{K}_{M / k} / K_{M / k}^{*}\right)$ have been studied in [10]. For the sake of simplicity and of later use, we treate here only the case where $M$ is a ray class field of $K \bmod . \mathfrak{⿰}, \widetilde{\mho}$ being any divisor of $K$.

For a finite or infinite prime $\mathfrak{P}$ of $K$ we denote by $K_{\mathfrak{B}}$ the local completion of $K$, by $J_{K}$ the idele group of $K$, and by $K^{\times}$the multiplicative group of non-zero elements of $K$. We embed $K_{\mathfrak{F}}$ and $K^{\times}$in $J_{K}$ in usual manner. Denote further by $U_{\Re}(r)$ the group of units $u$ of $K_{\mathfrak{B}}$ such that $u \equiv 1 \bmod$. $\mathfrak{P}^{r}$, where $r$ is a non-negative integer. When $\mathfrak{P}$ is an infinite prime, we take $r=0$ or 1 and by $u \equiv 1 \bmod$. $\beta$ mean that $u$ is a positive unit or any unit according as $\mathfrak{B}$ is real or imaginary. For a divisor $\mathfrak{F}=\prod_{\mathfrak{\beta}} \mathfrak{P}^{r}$ of $K$, set $U_{K}(\mathfrak{F})=\prod_{\Re} U_{\Re}\left(r_{\Re}\right)$.

Now let $M$ be a ray class field of $K \bmod$. $\preccurlyeq$. Then since $K^{\times} N_{M / K} J_{M}$ $=K^{\times} U_{K}(\mathfrak{F})$, the following propositions imply immediately from Proposition 1,2 and 3 of [10].

Proposition 4.1. $G\left(K_{M / k}^{*} / K\right) \cong k^{\times} N_{K / k} J_{K} / k^{\times} N_{K / k} U_{K}(\mathfrak{F})$.

Proposition 4.2.

$$
G\left(\hat{K}_{M / k} / K_{M / k}^{*}\right) \cong\left(k^{\times} \cap N_{K / k} J_{K}\right) / N_{K / k} K^{\times}\left(E_{k} \cap N_{K / k} U_{K}(\widetilde{\jmath})\right),
$$

where $E_{k}$ is the group of units of $k$.

Proposition 4.3. Notation $K, k$ and $M$ being as above, let $L$ be a subfield of $M$ which contains $K_{M / k}^{*}$. Then

$$
G\left(\hat{L}_{M / k} / L\right) \cong \frac{k^{\times} \cap N_{L / k} J_{L}}{N_{K / k}\left(K^{\times} \cap N_{L / K} J_{L}\right) \cdot\left(E_{k} \cap N_{K / k} U_{K}(\widetilde{\mho})\right)}
$$


Moreover by $[10, \S 3]$ we have

Proposition 4.4. Notation being as Proposition 4.3, assume further $E_{k} \cap N_{K / k} U_{K}(\mathfrak{F})=1$. Let $K$ and $L$ be normal over $k$ with Galois groups $g$ and $G$ respectively, and let $H$ be the Galois group of $L$ over $K$. Then

$$
\begin{aligned}
& G\left(\hat{L}_{M / k} / L\right) \cong H^{-3}(G, Z) /\left(\operatorname{cor}_{H, G} H^{-3}(H, Z)+\sum_{\nu} \operatorname{cor}_{G_{\nu}, G} H^{-3}\left(G_{\nu}, Z\right)\right), \\
& G\left(\hat{K}_{M / k} / K_{M / k}^{*}\right) \cong H^{-3}(g, Z) /\left(\sum_{\nu} \operatorname{cor}_{g_{\nu}, g} H^{-3}\left(g_{\nu}, Z\right)\right)
\end{aligned}
$$

where $G_{\nu}$ and $g_{\nu}$ are decomposition groups in $L / k$ resp. $K / k$ of an arbitrarily fixed prime divisor in $L$ resp. in $K$ of a prime $\mathfrak{p}_{\nu}$ of $k$.

\section{§5. Prime decomposition criteria}

5.1. As an application of the previous sections $\S 1$ and $\S 4$, we shall have some criteria of the prime decomposition in certain non-abelian extensions over $\boldsymbol{Q}$. This kind of criteria have been treated formerly by L. Rédei [20], S. Kuroda [18], A. Fröhlich [4] and [6] and Y. Furuta [7], and recently by E. Brown [1], K. Burde [2] and P. Kaplan [16].

Proposition 5.1. Let $K$ be a cyclic extension of an algebraic number field $k$ with Galois group $g$, which is generated by $\sigma$. Let $M$ be an abelian extension of $K$ which is normal over $k$. Let $L$ be an intermediate field between $M$ and $K$, and abelian over $k$ with Galois group G. Then $K_{M / k}^{(i+1)} \supset L_{M / k}^{(i)} \supset K_{M / k}^{(i)}$. Let further $\mathfrak{D}=\mathfrak{D}(M / K)$ be the ideal class group of $K$ corresponding to the class field $M$ over $K$, and denote by $C(\mathfrak{A})$ the class of $\mathfrak{D}$ represented by an ideal $\mathfrak{U}$ of $K$. Then the notation $\mathfrak{S}_{\mathfrak{L}}(L / K)$ and $\AA^{(i)}=\Re^{(i)}(\mathfrak{D})$ being as in (1) and (3), we have

$$
G\left(L_{M / k}^{(i)} / K_{M / k}^{(i)}\right) \cong \mathfrak{D} / C(\mathfrak{E}(L / K)) \Re^{(i)} \cong G\left(K_{i} / K\right),
$$

where $K_{i}$ is the largest extension of $K$ in $L$ such that every prime $\mathfrak{p}$ of $K$ is completely decomposed in $K_{i}$ when $\mathfrak{p}$ is contained in a class of $\AA^{(i)}$.

Moreover let $\mathfrak{A}$ be an ideal of $K$ which is the norm of an ideal $\mathfrak{A}^{(i)}$ of $K_{M / k}^{(i)}$ and prime to the conductor $\mathfrak{\mho}(M / K)$, and $\mathfrak{B}$ be an ideal of $K$ such that $\mathfrak{B}^{(\sigma-1)^{i}} \equiv \mathfrak{A} \bmod . \mathfrak{S C}_{\mathcal{C}}(M / K)$. Let $\sigma$ and $\tau$ be elements of $G\left(L_{M / k}^{(i)} / K_{M / k}^{(i)}\right)$ and $G\left(K_{i} / K\right)$ which correspond to $\mathfrak{U}^{(i)}$ and $\mathfrak{B}$ respectively by Artin's reciprocity map. Then $\sigma$ and $\tau$ correspond each other by the above isomorphism. 
Proof. It follows from the translation theorem of class field theory that the subgroup of () corresponding to $L_{M / k}^{(i)}$ over $K$ is equal to $C\left(N_{L / K} I_{G}^{i}\left(\widetilde{\Im}_{L}\right)\right)=C\left(N_{L / K}\left(\widetilde{\Im}_{L}\right)^{(\sigma-1)^{i}}\right)=C\left(\mathfrak{S}_{\mathcal{C}}(L / K)^{(\sigma-1)^{i}}\right)$, where $\widetilde{\Im}_{L}$ is the group of ideals of $L$ which is prime to the conductor $\mathfrak{F}(M / L)$. Clearly $C\left(S_{\mathcal{C}}(L / K)^{(\sigma-1)^{i}}\right) \subset \mathfrak{D}^{(\sigma-1)^{i}}$. Hence $L_{M / k}^{(i)} \supset K_{M / k}^{(i)}$ and we have $G\left(L_{M / k}^{(i)} / K_{M / k}^{(i)}\right) \cong$ $\mathfrak{D}^{(\sigma-1)^{i}} / C\left(\mathscr{S}(L / K)^{(\sigma-1)^{i}}\right)$. Moreover since $L \subset K_{M / k}^{*}=K_{M / k}^{(1)}, C\left(\mathfrak{S}_{\mathcal{C}}(L / K)\right) \supset \mathfrak{D}^{\sigma-1}$. Hence $C\left(S_{\mathcal{C}}(L / K)^{(\sigma-1) i}\right) \supset \mathfrak{D}^{(\sigma-1)^{i+1}}$ and $L_{M / k}^{(i)} \subset K_{M / k}^{(i+1)}$. Now the isomorphism of (1) in $\S 1$ implies immediately the proposition.

5.2. By considering the special case where $K$ is a quadratic field, $L$ is a biquadratic field and $i=1$, we have some criteria for the prime decomposition in non-abelian normal fields of degree 8 .

Proposition 5.2. Let $\boldsymbol{Q}\left(\sqrt{d_{1}}\right)$ and $\boldsymbol{Q}\left(\sqrt{d_{2}}\right)$ be two quadratic fields, $L$ be their composite, and $K=Q(\sqrt{d})$ be the intermediate field of $L$ over $\boldsymbol{Q}$ distinct from $\boldsymbol{Q}\left(\sqrt{d_{1}}\right)$ and $\boldsymbol{Q}\left(\sqrt{d_{2}}\right)$. Let $\Lambda$ be a quadratic extension of $L$ which is normal and non-abelian over $\boldsymbol{Q}$. Let further $M$ be $a$ normal extension over $\boldsymbol{Q}$, abelian over $K$ and contains $\Lambda$. Denote $M_{0}$ the largest subfield of $M$ which is abelian over $\boldsymbol{Q}$. Then notation being as in $\S 1$, every prime of $K$ which represents a class of $\AA^{(1)}(D(M / K)$ ) is decomposed completely in $L$. Moreover let $\mathfrak{p}$ be a prime ideal of $L$ which is degree 1 over $\boldsymbol{Q}$ and is decomposed completely in $M_{0}$. Then we have

$$
(\Lambda / L, \mathfrak{p})=(L / Q, q),
$$

where $q$ is any rational prime such that $4 p q^{2}=x^{2}-y^{2} D, \frac{1}{2}(x+y \sqrt{D})$ $\equiv q \bmod . \mathfrak{F}(M / K)$ for $p=N_{L / Q} \mathfrak{p}$, some integers $x, y$ and the discriminant $D$ of $K / \boldsymbol{Q}$.

Proof. We have $L_{M / Q}^{(1)} \supset K_{M / Q}^{(1)}=K_{M / Q}^{*}=L_{M / Q}^{*}=M_{0}$, and Proposition 5.1 implies

$$
G\left(L_{M / Q}^{(1)} / K_{M / Q}^{(1)}\right) \cong \Im / C(\mathscr{S}(L / K)) \Re^{(1)},
$$

which is isomorphic to a subgroup of $G(L / K)$. Moreover $L_{M / Q}^{(1)}$ is quadratic over $K_{M / Q}^{(1)}$ if and only if every prime of $K$ which represents a class of of $\AA^{(1)}$ is decomposed completely in $L$. On the other hand since $\Lambda$ is quadratic over $L, \Lambda$ is central over $L$ with respect to $\boldsymbol{Q}$. Moreover since $\Lambda$ is non-abelian over $\boldsymbol{Q}$, we have $L_{M / Q}^{(1)}=\Lambda M_{0} \supseteq M_{0}=K_{M / Q}^{(1)}$. Thus our first assertion of the proposition is proved.

Let $\mathfrak{p}=N_{M_{0} / L} \mathfrak{P}$ for a prime $\mathfrak{P}$ of $M_{0}$. Then Proposition 5.1 implies 


$$
(\Lambda / L, \mathfrak{p})=\left(\Lambda M_{0} / M_{0}, \mathfrak{P}\right)=(L / K, \mathfrak{q}),
$$

where $\mathfrak{q}^{\sigma-1} \equiv N_{M_{0} / K} \mathfrak{P} \bmod H(M / K)$, $\sigma$ being the generator of $G(K / \boldsymbol{Q})$. Now the last assertion of the proposition follows from $\S 1.3$.

Proposition 5.3. Let $K, L$ and $\Lambda$ be as in Proposition 5.2. and assume that $\Lambda$ is an EL-genus extension of $L$ with respect to $Q$. Then every prime of $K$ which is ramified over $\boldsymbol{Q}$ is decomposed completely in L. Moreover let $K^{\#}$ be the absolute class field of $K$ in narrow sense and $K_{\#}^{*}$ be the genus field of $K$ in $K^{\#}$ with respect to $\boldsymbol{Q}$. Let $\mathfrak{p}$ be a prime of $L$ which is of degree 1 over $\boldsymbol{Q}$ and decomposed completely ${ }^{13}$ in $K_{\#}^{*}$. Then there exists an abelian extension $A$ of $\boldsymbol{Q}$ such that

$$
(\Lambda / L, \mathfrak{p})=(L / \boldsymbol{Q}, q)(A / \boldsymbol{Q}, p),
$$

where $p=N_{L / Q} \mathfrak{p}$ and $4 p q^{2}=x^{2}-y^{2} D$ by the discriminant $D$ of $K / \boldsymbol{Q}$ and rational integers $x$ and $y$ such that $(q, x, y)=1$.

Proof. It is well known that $L$ is contained in $K^{\sharp}$. We apply Proposition 5.2 to the case where $M$ contains $K^{\#}$. Let $g$ be the Galois group of $L$ over $\boldsymbol{Q}$, and denote by $\hat{L}$ resp. $L^{*}$ the $E L$-genus central extension resp. the genus field in $K^{\#}$ with respect to $\boldsymbol{Q}$. Then since

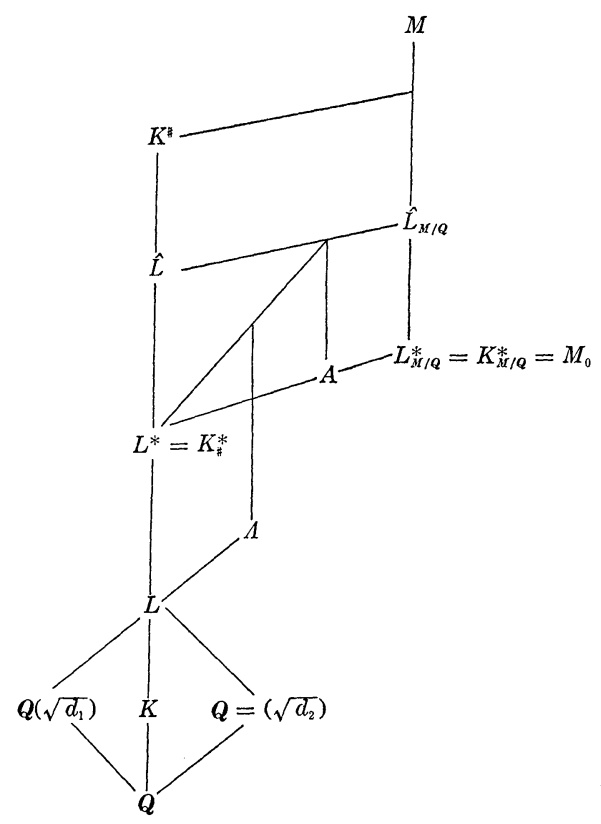

13) This is characterized by using "Auflösung" character. 


$$
E_{\boldsymbol{Q}} \cap N_{L / \boldsymbol{Q}} U_{L}(\mathfrak{\mho}(M / L)) \subseteq E_{\boldsymbol{Q}} \cap N_{L / \boldsymbol{Q}} U_{L}\left(\widetilde{\mho}\left(K^{\sharp} / L\right)\right)=1,
$$

Proposition 4.4 implies

$$
G\left(\hat{L}_{M / Q} / L_{M / Q}^{*}\right) \cong G\left(\hat{L} / L^{*}\right) \cong H^{-3}(g, Z) / \sum_{\nu} \operatorname{cor}_{g_{\nu}, g} H^{-3}\left(g_{\nu}, Z\right),
$$

where $g_{\nu}$ runs over the decomposition groups of primes in $L$ ramified over $\boldsymbol{Q}$. Since $\Lambda$ is contained in $\hat{L}_{M / \boldsymbol{Q}}$ and $\Lambda$ is non-abelian over $\boldsymbol{Q}, \hat{L}_{M / \boldsymbol{Q}}$ is quadratic over $L_{M / Q}^{*}=K_{M / Q}^{*}$. Hence (19) implies $g_{\nu} \subseteq g$ for every $\nu$, which implies the first assertion of the proposition. Now we have $\hat{L}_{M / Q}$ $=A L_{M / Q}^{*}=\hat{L} L_{M / Q}^{*}$. Hence there exists a quadratic extension $A$ of $K_{\#}^{*}$ such that $A$ is contained in $L_{M / Q}^{*}$ and $\hat{L} A=\hat{L} A$. Let $\mathfrak{p}$ be a norm of a prime $\mathfrak{\beta}$ of $K_{\#}^{*}=L^{*}$ to $L$. Then

$$
(\Lambda / L, \mathfrak{p})=\left(\Lambda L^{*} / L^{*}, \mathfrak{P}\right)=\left(\hat{L} / L^{*}, \mathfrak{P}\right)\left(A / L^{*}, \mathfrak{P}\right) .
$$

Apply Proposition 5.1 to the case where $k=\boldsymbol{Q}, \quad M=K^{\#}$ and $i=1$. Then since $L_{M / k}^{(1)}=\hat{L}$ and $K_{M / k}^{(1)}=K_{\sharp}^{*}=L^{*}$, we have $\left(\hat{L} / L^{*}, \mathfrak{P}\right)=(L / K, \mathfrak{q})$, where $\mathfrak{q}^{\sigma-1}=\mathfrak{p}_{1} \alpha, \mathfrak{p}_{1}=N_{L^{*} / K} \mathfrak{P}$ and $\alpha$ is a totally positive element of $K$. Moreover it follows from $\S 1.3$ that $(L / K, \mathfrak{q})=(L / Q, q)$. Clearly $\left(\Lambda / L^{*}, \mathfrak{\beta}\right)$ $=(A / Q, p)$. Thus the proposition is proved.

5.3. We can have a reciprocity law of the restricted biquadratic residue symbol, by applying Proposition 5.2 and 5.3 to the special case $d_{2}=-1$.

Let $q$ be a rational prime such that $q \equiv 1 \bmod .4$, and put $K=$ $\boldsymbol{Q}(\sqrt{-q})$. Let $L$ be the composite of $K$ and $Q(\sqrt{-1})$ and let $B$ be the subfield of degree 4 of the ray class field over $\boldsymbol{Q}$ mod. $q$. Let further $\Lambda$ be the subfield of $L(\sqrt[4]{q}) B$ over $L$ of degree 2 distinct from $L B$ and $L(\sqrt[4]{q})$. Then it follows from [7, Theorem 2] that if $p$ is a rational prime such that $(q / p)=1$, then

$$
\left(\frac{q}{p}\right)_{4}\left(\frac{p}{q}\right)_{4}=(\Lambda / L, \mathfrak{p}),
$$

where $(-)_{4}$ is the forth power residue symbol in $\boldsymbol{Q}$ and $\mathfrak{p}$ is a prime divisor of $p$ in $L$.

Now let us apply Proposition 5.3 to the right hand side of (20).

Proposition 5.4. Let $p$ and $q$ be rational primes such that $p, q \equiv 1$ 
$\bmod .4$ and $(q / p)=1$. Then we have $e^{14)}$

$$
\left(\frac{q}{p}\right)_{4}\left(\frac{p}{q}\right)_{4}=\left(\frac{p_{1}}{q}\right)=\left(\frac{-1}{p_{1}}\right)
$$

where $p_{1}$ is any rational prime which is a primitive solution ${ }^{15)} v$ of the Diophantine equation $p v^{2}=x^{2}+y^{2} q$ or $p v^{2}=x^{2}+4 y^{2} q$ according as $q \equiv 1$ or $\not \equiv 1$ mod. 8 .

Proof. Let notation $L^{*}, \hat{L}, K_{*}^{*}, M$ and $M_{0}$ be as in Proposition 5.3 and its proof. Then $L=L^{*}=K_{\sharp}^{*}$. Moreover $\hat{L} \supseteq L^{*}$ if and only if the class number of $K$ is divisible by 4 , which is equivalent ${ }^{16)}$ to $q \equiv 1 \bmod$. 8 .

(i) Assume $q \equiv 1 \bmod$. 8. Then $\hat{L} \supsetneq L^{*}$, which implies $\hat{L}_{M / Q} \supsetneq L_{M / Q}^{*}$ by (19) and $\Lambda M_{0}=\hat{L}_{M / Q}$. Since prime divisors of 2 in $\boldsymbol{Q}(\sqrt[4]{q})$ are unramified over $\boldsymbol{Q}(\sqrt{q})$, we choose $M$ to be the ray class field over $K$ mod. $q$. Then $L B$ is the unique quadratic extension of $L^{*}=L$ in $M_{0}$. Since $\hat{L} \supseteq L=L^{*}$, it follows from the definition of $\Lambda$ that $\Lambda$ is equal to $\hat{L}$ and the field $A$ in Proposition 5.3 is equal to $L$. Now Proposition 5.3 and (20) imply the present proposition in the case $q \equiv 1 \bmod .8$.

(ii) Assume $q \neq 1 \bmod$. 8. Then $\hat{L}=L^{*}=L$ as seen above. Moreover we can see ${ }^{17)}$ that prime divisors of 2 in $\boldsymbol{Q}(\sqrt[4]{q})$ are ramified over $\boldsymbol{Q}(\sqrt{q})$. This implies that the only prime divisors of 2 are ramified in $\Lambda$ over $L$. Thus we choose $M$ to be the ray class field over $K \bmod 2$. We note that $\Lambda$ is not an $E L$-genus extension of $K$ with respect to $\boldsymbol{Q}$, for otherwise the above equality $\hat{L}=L^{*}$ contradicts to (19). Now the proposition is followed from Proposition 5.2 and (20).

5.4. The following table is a numerical example for Proposition 5.4 when $p$ and $q$ are smaller than 53 and $p_{1}$ is smaller than 19.

The numbers $n$ for $(q / p)_{4}$ means $n^{4} \equiv q \bmod . p$, and the symbol $\Delta$ means $(q / p)_{4}=-1$.

14) This reciprocity has other expression from that of E. Brown [1], K. Burde [2] or P. Kaplan [16]. The latters follow from S. Kuroda [18]. See also A. Fröhlich [16].

15) There exists such $p_{1}$ except the case $p=x^{2}+y^{2} q$ by some integers $x$ and $y$. Cf. §1.3.

16) See for instance H. Hasse [14].

17) See for instance Hasse's Bericht Ia, §11, Satz 9 and II, §9, XI. 


\begin{tabular}{|c|c|c|c|c|c|c|c|c|}
\hline$p$ & $q$ & $4 q$ & $v=p_{1}$ & $x$ & $y$ & $(q / p)_{4}$ & $(p / q)_{4}$ & $\left(p_{1} / q\right)$ \\
\hline \multirow{2}{*}{5} & \multirow{2}{*}{29} & \multirow{2}{*}{116} & 5 & 3 & 1 & \multirow{2}{*}{$\triangle$} & \multirow{2}{*}{$\triangle$} & 1 \\
\hline & & & 13 & 21 & 1 & & & 1 \\
\hline \multirow{4}{*}{5} & \multirow{4}{*}{41} & & 3 & 2 & 1 & \multirow{4}{*}{1} & \multirow{4}{*}{$\triangle$} & -1 \\
\hline & & & 7 & 9 & 2 & & & -1 \\
\hline & & & 11 & 21 & 2 & & & -1 \\
\hline & & & 19 & 42 & 1 & & & -1 \\
\hline \multirow{3}{*}{13} & \multirow{3}{*}{17} & & 3 & 7 & 2 & \multirow{3}{*}{$\triangle$} & \multirow{3}{*}{3} & -1 \\
\hline & & & 7 & 5 & 6 & & & -1 \\
\hline & & & 11 & 31 & 6 & & & -1 \\
\hline \multirow{2}{*}{13} & \multirow{2}{*}{53} & \multirow{2}{*}{212} & 13 & 17 & 3 & \multirow{2}{*}{1} & \multirow{2}{*}{11} & 1 \\
\hline & & & 17 & 43 & 3 & & & 1 \\
\hline \multirow{4}{*}{17} & \multirow{4}{*}{13} & \multirow{4}{*}{52} & 2 & 4 & 1 & \multirow{4}{*}{3} & \multirow{4}{*}{$\triangle$} & -1 \\
\hline & & & 7 & 1 & 4 & & & -1 \\
\hline & & & 11 & 43 & 2 & & & -1 \\
\hline & & & 19 & 77 & 2 & & & -1 \\
\hline \multirow{2}{*}{17} & \multirow{2}{*}{53} & \multirow{2}{*}{212} & 13 & 45 & 2 & \multirow{2}{*}{$\triangle$} & & 1 \\
\hline & & & 17 & 39 & 4 & & $\Delta$ & 1 \\
\hline 29 & 5 & 20 & 1 & 3 & 1 & & & \\
\hline & & & 3 & 7 & 1 & & & -1 \\
\hline 29 & 53 & 212 & 19 & 9 & 7 & 4 & $\Delta$ & -1 \\
\hline & & & 3 & 13 & 2 & & & -1 \\
\hline 97 & 11 & & 7 & 38 & 3 & A & 0 & -1 \\
\hline 37 & 41 & & 11 & 34 & 9 & $\Delta$ & 8 & -1 \\
\hline & & & 19 & 109 & 6 & & & -1 \\
\hline & 52 & ת19 & 3 & 11 & 1 & O & 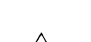 & -1 \\
\hline 37 & Do & 212 & 19 & 107 & 3 & 2 & $\Delta$ & -1 \\
\hline & & & 2 & 12 & 1 & & & -1 \\
\hline 41 & 5 & 20 & 3 & 17 & 2 & $\triangle$ & 1 & -1 \\
\hline & & & 7 & 27 & 8 & & & -1 \\
\hline & & & 2 & 4 & 1 & & & -1 \\
\hline 41 & 37 & 148 & 19 & 73 & 8 & 8 & $\triangle$ & -1 \\
\hline 53 & 17 & & 1 & 6 & 1 & & & \\
\hline 53 & 29 & 116 & 3 & 19 & 1 & $\triangle$ & 4 & -1 \\
\hline 53 & 37 & 148 & 2 & 8 & 1 & $\hat{0}$ & $?$ & -1 \\
\hline 00 & Ji & 140 & 19 & 109 & 7 & $\Delta$ & 2 & -1 \\
\hline
\end{tabular}




\section{REFERENCES}

[1] E. Brown, Biquadratic reciprocity laws, Proc. Amer. Math. Soc., 37 (1973), 374376.

[2] K. Burde, Ein rationales biquadratisches Reziprozitätsgesetz, J. reine und angew. Math., 235 (1969), 175-184.

[ 3 ] A. Fröhlich, On the absolute class-group of abelian fields, (I), (II), J. London Math. Soc., 29 (1954), 211-217; 30 (1955), 72-80.

[4] A. Fröhlich, The restricted biquadratic residue symbol, Proc. London Math. Soc., 9 (1959), 189-207.

[5] A. Fröhlich, The genus field and genus group in finite number fields I, II, Mathematika, 6 (1959), 40-46, 142-146.

[6] A. Fröhlich, A prime decomposition symbol for certain non Abelian number fields, Acta Sci. Math., 21 (1960), 229-246.

[7] Y. Furuta, A reciprocity law of the power residue symbol, J. Math. Soc. Japan, 10 (1958), 46-54.

[ 8 ] Y. Furuta, The genus field and genus number in algebraic number fields, Nagoya Math. J., 29 (1967), 281-285.

[ 9 ] Y. Furuta, On class field towers and the rank of ideal class group, Nagoya Math. J., 48 (1972), 147-157.

[10] Y. Furuta, On nilpotent factors of congruent ideal class groups of Galois extensions, Nagoya Math. J., 62 (1976), 13-28.

[11] L. J. Goldstein, On prime discriminants, Nagoya Math. J., 45 (1972), 119-127.

[12] G. Gras, Sur le $\ell$-classes d'ideaux dans les extensions cycliques relatives de degré premier $\ell$, Ann. Inst. Fourier, Grenoble, 23, 3 (1973) ,1-48; 23, 4 (1973), 1-44.

[13] H. Hasse, Zur Geschlechtertheorie in quadratischen Zahlkörper, J. Math. Soc. Japan, 3 (1951), 45-51.

[14] H. Hasse, Über die Teilbarkeit durch $2^{3}$ der Klassenzahl der quadratischen Zahlkörper mit genau zwei verschiedenen Diskriminantenprimteilern, Math. Nachr., 46 (1970), 61-70.

[15] E. Inaba, Über die Struktur der $\ell$-klassengruppe zyklischer Zahlkörper von Primzahlgrad $\ell$, J. Fac. Sci. Univ. Tokyo, 4 (1940), 61-115.

[16] P. Kaplan, Divisibilité par 8 du nombre des classes des corps quadratiques réels dont le 2-sous-groupe des classes est cyclique, C. R. Acad. Sci. Paris, 275 (1972), $887-890$.

[17] H. Koch, Über die 2-Komponente der Klassengruppe quadratischer Zahlkörper mit zwei Diskriminantenteilern, Math. Nachr., 54 (1972), 309-333.

[18] S. Kuroda, Über die Zerlegung rationaler Primzahlen in gewissen nicht-abelschen galoisschen Körpern, J. Math. Soc. Japan, 3 (1951), 148-156.

[19] H. W. Leopoldt, Zur Geschlechtertheorie in abelschen Zahlkörpern, Math. Nachr., 9 (1953), 351-362.

[20] L. Rédei, Über die Grundeinheit und die durch 8 teilbaren Invarianten der absoluten Klassengruppe im quadratischen Zahlkörper, J. reine und angew. Math., 171 (1934), 131-148.

\section{Kanazawa University}

